Supporting Information for

\title{
Coexistence of superconductivity and nontrivial band topology in monolayered cobalt pnictides on $\mathrm{SrTiO}_{3}$
}

Jiaqing Gao, Wenjun Ding, Shunhong Zhang, Zhenyu Zhang ${ }^{*}$, Ping Cui ${ }^{*}$

International Center for Quantum Design of Functional Materials (ICQD), Hefei National Laboratory for Physical Sciences at Microscale (HFNL), and CAS Center for Excellence in Quantum Information and Quantum Physics, University of Science and Technology of China, Hefei, Anhui 230026, China

*Corresponding authors. Emails: zhangzy@ustc.edu.cn (Z.Y.Z.); cuipg@ustc.edu.cn (P.C.)

\section{Methods and computational details}

The first-principles calculations were based on density functional theory (DFT). Structural relaxations and calculations of the electronic properties were performed by using the Vienna Ab initio Simulation Package (VASP) ${ }^{1}$ adopting the projector augmented wave $(\mathrm{PAW})^{2}$ for describing the valence electrons, generalized gradient approximation (GGA) of Perdew-Burke-Ernzerhof $(\mathrm{PBE})^{3}$ for the exchange correlation potentials, and a $21 \times 21$ $\times 1$ Monkhorst-Pack $k$-point mesh for the Brillouin zone sampling. The kinetic energy cutoff of the plane-wave basis was chosen to be $400 \mathrm{eV}$. The electronic minimizations were performed with an energy tolerance of $10^{-6} \mathrm{eV}$, and optimized atomic structures were achieved when forces on all the atoms were smaller than $0.01 \mathrm{eV} / \AA$. To prevent the interactions between adjacent supercells, we set a vacuum layer perpendicular to the $\operatorname{Co} X$ 
sheet of larger than $18 \AA$. The semi-empirical DFT-D3 method ${ }^{4}$ was chosen for the van der Waals corrections, and the electric field induced by asymmetric slab relaxation was compensated by a dipole correction ${ }^{5}$ for the $\mathrm{Co} X / \mathrm{STO}$ systems.

We employed the Quantum ESPRESSO package $^{6}$ to explore the superconducting properties of $\operatorname{Co} X$ monolayers, The electron-phonon coupling (EPC) strength $\lambda$ can be calculated from the Eliashberg function $\alpha^{2} F(\omega)$ as: $:^{7,8}$

$$
\lambda=2 \int_{0}^{\infty} \frac{\alpha^{2} F(\omega)}{\omega} d \omega,
$$

where $\omega$ is the phonon frequency. We then estimate the superconducting transition temperature $\left(T_{\mathrm{c}}\right)$ by using the McMillan-Allen-Dynes parametrized equations: ${ }^{7,8}$

$$
\begin{gathered}
k_{\mathrm{B}} T_{\mathrm{c}}=\frac{\hbar \omega_{\log }}{1.2} \exp \left[-\frac{1.04(1+\lambda)}{\lambda-\mu^{*}(1+0.62 \lambda)}\right], \\
\omega_{\log }=\exp \left[\frac{2}{\lambda} \int_{0}^{\infty} d \omega \frac{\alpha^{2} F(\omega)}{\omega} \log \omega\right],
\end{gathered}
$$

where $\omega_{\log }$ is the logarithmic average of the phonon frequencies, and $\mu^{*}$ is a parameter describing the Coulomb repulsion, which usually takes values in the range of $0.1-0.2 .^{9}$ The dynamical matrices and EPC matrices were computed with $a$ k-mesh of $9 \times 9 \times 1$ and a $q$-mesh of $3 \times 3 \times 1$, respectively. The maximally localized Wannier functions (MLWFs) were constructed by the Wannier90 package, ${ }^{10}$ and the $\mathbf{Z}_{2}$ invariant and edge-state calculations were done using the WannierTools package. ${ }^{11}$ 


\section{Additional figures}
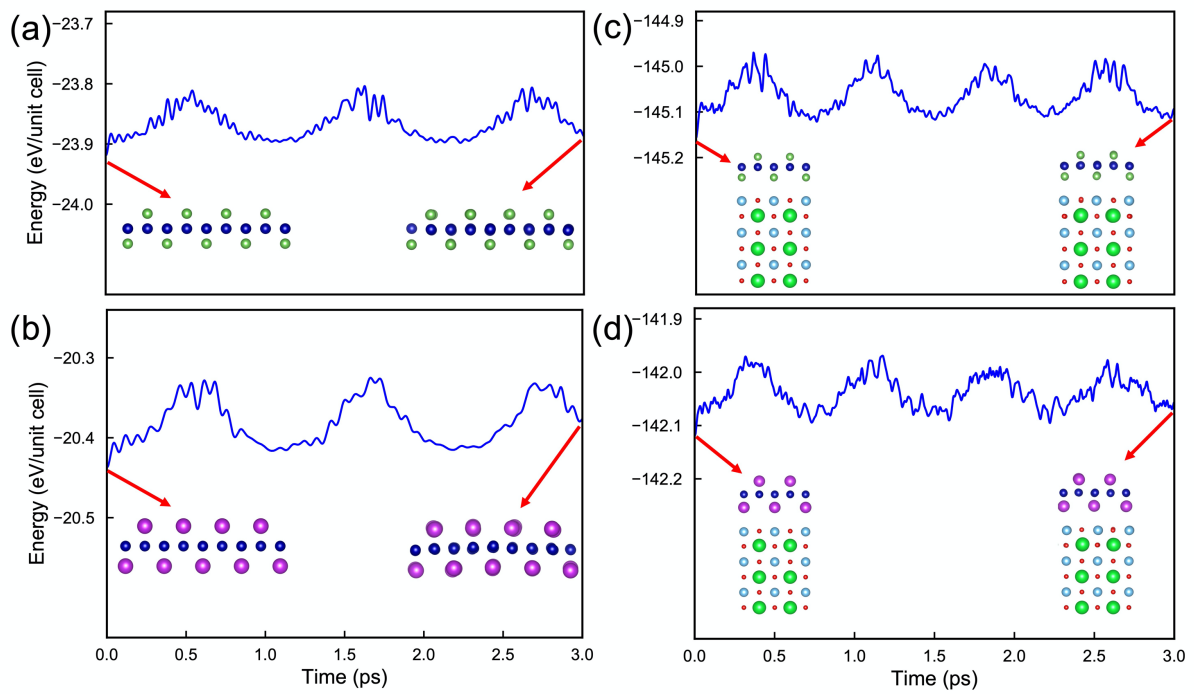

Figure S1. Ab initio molecular dynamics (AIMD) simulations. Evolutions of the total energies of (a) a freestanding CoAs monolayer, (b) a freestanding CoBi monolayer, (c) $\mathrm{CoAs} / \mathrm{STO}$, and (d) $\mathrm{CoBi} / \mathrm{STO}$ during the AIMD simulations at $100 \mathrm{~K}$ and up to $3 \mathrm{ps}$. The insets display the side views of the atomic structures at the beginning and end of the simulations.

(a)
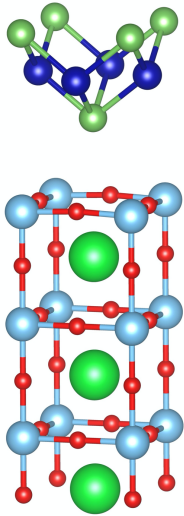

(b)
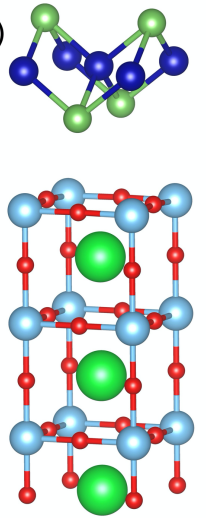

(c)
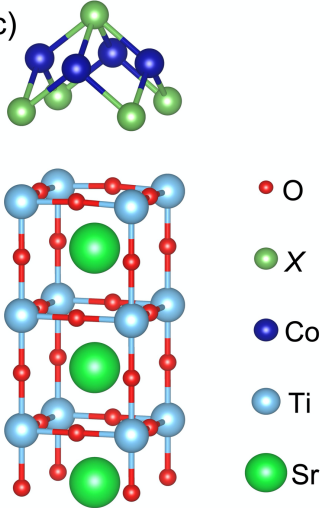

Figure S2. Three high-symmetry stacking configurations of $\mathrm{Co} X / \mathrm{STO}(001)$ with $X=\mathrm{As}$, Bi. (a) Bottom $X$ atoms located on the hollow site of the surface $\mathrm{TiO}_{2}$. (b) Bottom $X$ atoms located on top of surface oxygen atoms. (c) Bottom $X$ atoms located on top of surface Ti atoms. 

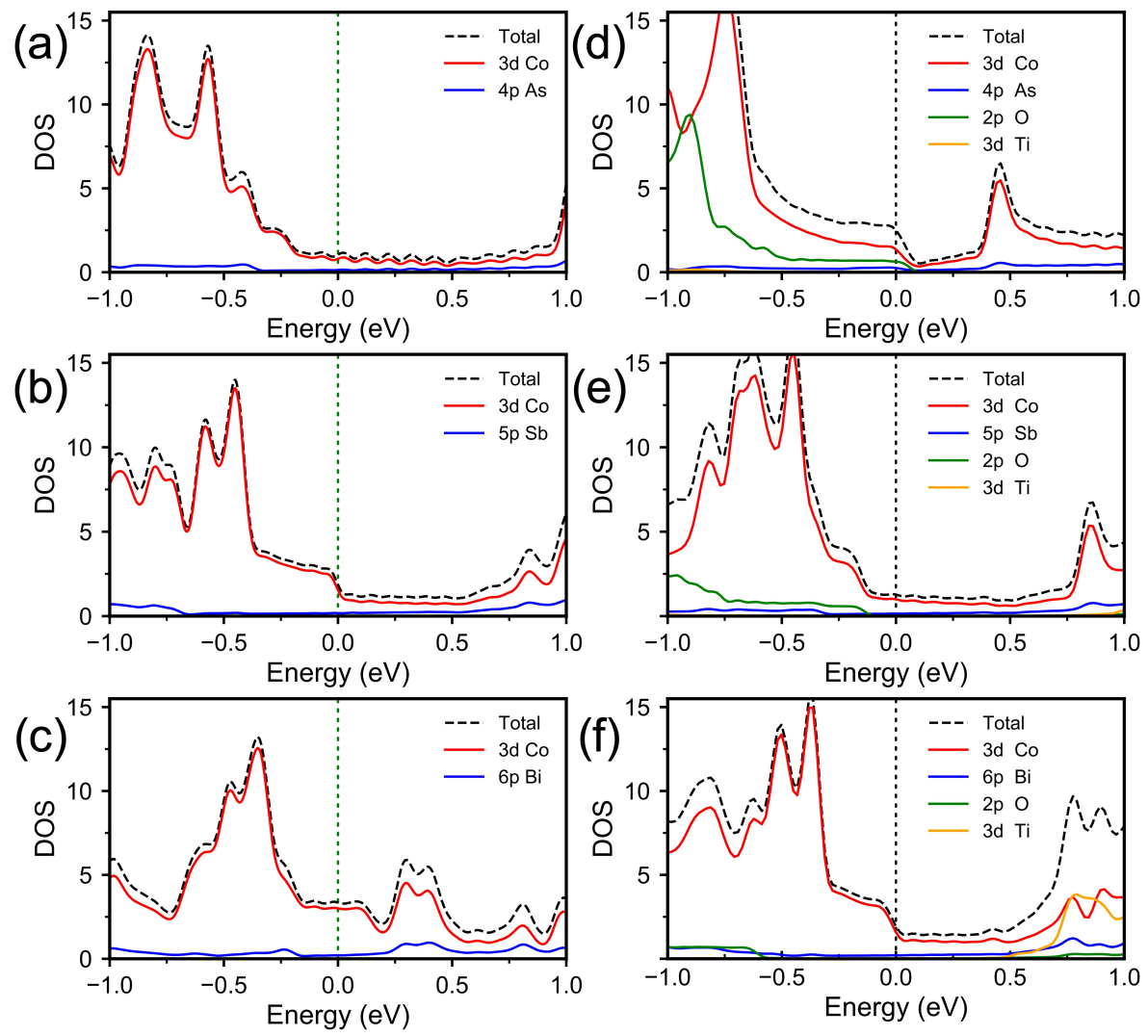

Figure S3. Projected density of states (DOS) of freestanding $\operatorname{Co} X$ monolayers and $\mathrm{CoX} / \mathrm{STO}$. (a) CoAs, (b) $\mathrm{CoSb}$, (c) CoBi, (d) CoAs/STO, (e) CoSb/STO, and (f) CoBi/STO. The Fermi levels are all set at zero. 

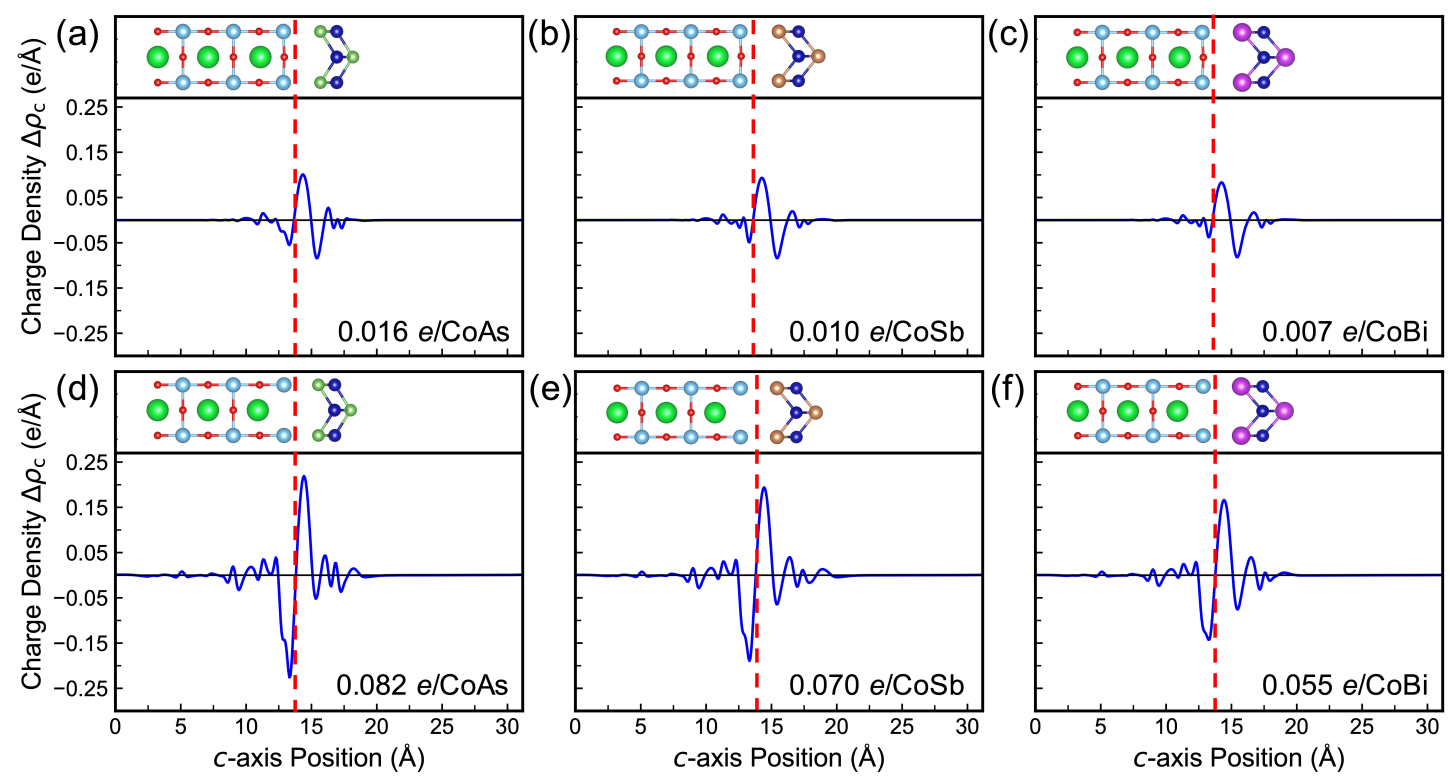

Figure S4. Charge transfer of CoX/STO. $(\mathrm{a}, \mathrm{b}, \mathrm{c})$ Charge density $\Delta \rho_{\mathrm{c}}$ of $\operatorname{Co} X / \mathrm{STO}$ with $X$ $=\mathrm{As}, \mathrm{Sb}, \mathrm{Bi}$, respectively. (d, e, f) Charge density $\Delta \rho_{\mathrm{c}}$ of surface-oxygen-deficient $\mathrm{Co} X / \mathrm{STO}$ with $X=\mathrm{As}, \mathrm{Sb}, \mathrm{Bi}$, respectively. The corresponding atomic structure for each system is shown on the top. The charge density $\Delta \rho_{\mathrm{c}}$ is defined as the plane-averaged charge density difference along the $c$ axis between the combined $\operatorname{CoX} / \mathrm{STO}$ and the summation of the isolate $\operatorname{Co} X$ monolayers and STO substrate. The amount of charge transfer can be obtained by integrating $\Delta \rho_{\mathrm{c}}$ within the area at the right side of the red dashed vertical line, namely, the region of the $\operatorname{Co} X$ overlayers. The amount of charge transfer for each system is indicated in the bottom right corner. 

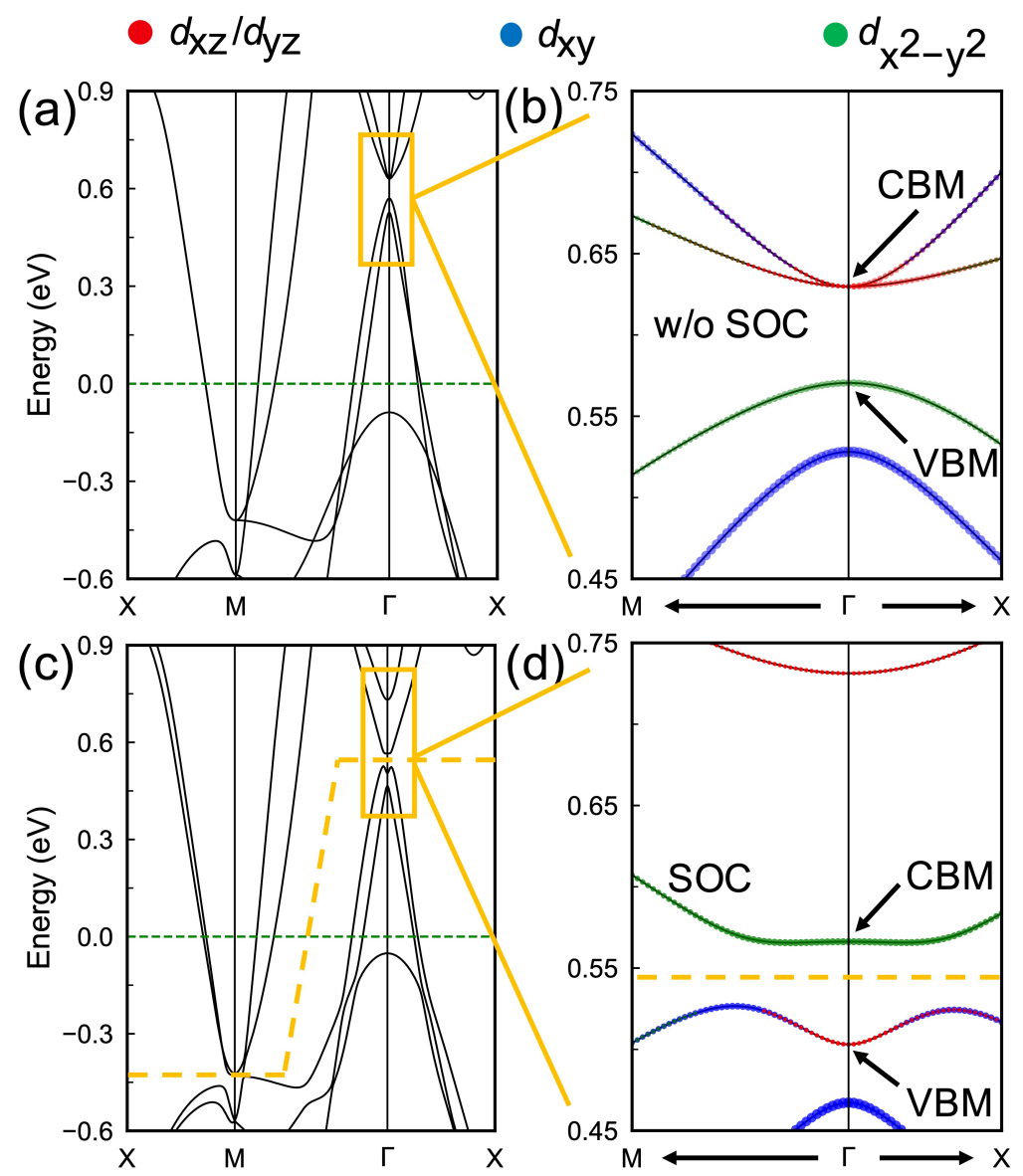

Figure S5. Electronic structures of a CoSb monolayer with a tensile strain $(a=3.780 \AA)$. Band structures (a) without and (c) with the SOC. (b), (d) Zoom-in views of the solid orange box areas in (a), (c), respectively. The radii of the red, blue, and green dots indicate the spectral weights of the Co- $d_{x z} / d_{y z}, d_{x y}$, and $d_{x^{2}-y^{2}}$ orbitals. The orange dashed lines correspond to the curved chemical potentials. The Fermi levels are all set at zero. When the lattice constant $a$ of a CoSb monolayer is increased to $3.780 \AA$, the band gap at the $\Gamma$ point is reduced to $59 \mathrm{meV}$ with the inclusion of the SOC. Without the SOC, the conduction band minimum (CBM) is mainly contributed by Co- $d_{x z} / d_{y z}$ and $\mathrm{Sb}-p_{x} / p_{y}$ orbitals, and the valence band maximum (VBM) is mainly contributed by Co- $d_{x^{2}-y^{2}}$ and $\mathrm{Sb}-p_{z}$ orbitals. When the SOC is included, a band inversion takes place at the $\Gamma$ point and the $\mathbf{Z}_{2}$ invariant is calculated to be 1 , indicating that the system is topologically nontrivial. 

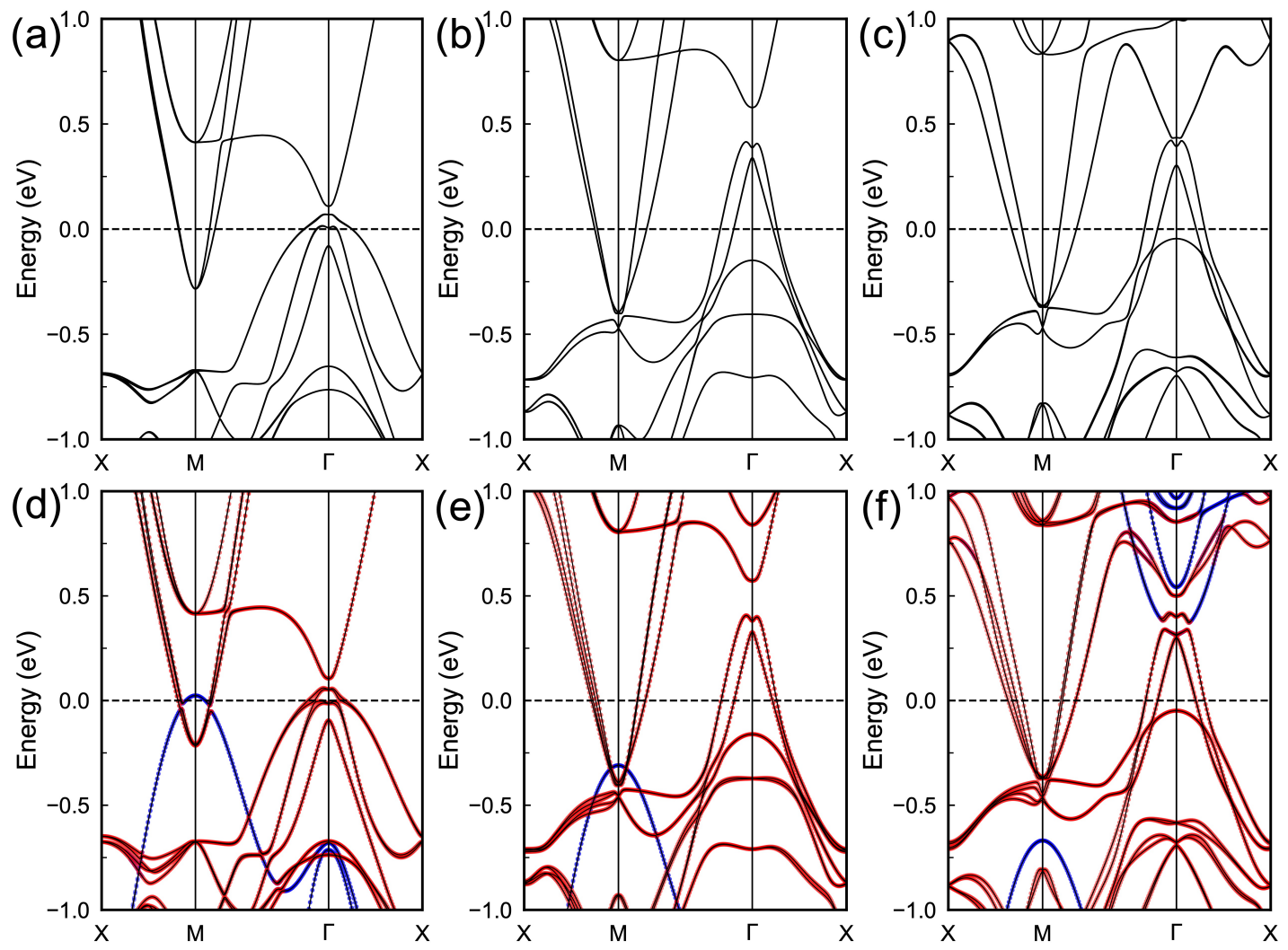

Figure S6. Electronic structures of $\operatorname{Co} X$ monolayers $(a=3.905 \AA)$ and $\operatorname{Co} X / \mathrm{STO}$ with the SOC. $(\mathrm{a}, \mathrm{b}, \mathrm{c})$ Band structures of CoAs, CoSb, and CoBi monolayers, respectively, with their atomic structures determined in the presence of the STO substrate. $(\mathrm{d}, \mathrm{e}, \mathrm{f})$ Band structures of CoAs on one STO layer, CoSb on one STO layer, and CoBi on two STO layers, respectively. The contributions from the $\operatorname{Co} X$ overlayers are indicated in red and the contributions from the STO are indicated in blue. The Fermi levels are all set at zero.

\section{References}

(1). Kresse, G.; Furthmüller, J. Efficient iterative schemes for $a b$ initio total-energy calculations using a plane-wave basis set. Phys. Rev. B 1996, 54, 11169.

(2). Blochl, P. E. Projector augmented-wave method. Phys. Rev. B 1994, 50, 17953-17979.

(3). Perdew, J. P.; Burke, K.; Ernzerhof, M. Generalized gradient approximation Mmade simple. Phys. Rev. Lett. 1996, 77, 3865. 
(4). Grimme, S.; Antony, J.; Ehrlich, S.; Krieg, H. A consistent and accurate ab initio parametrization of density functional dispersion correction (DFT-D) for the 94 elements H-Pu. J. Chem. Phys. 2010, 132, 154104.

(5). Neugebauer, J.; Scheffler, M. Adsorbate-substrate and adsorbate-adsorbate interactions of $\mathrm{Na}$ and $\mathrm{K}$ adlayers on $\mathrm{Al}(111)$. Phys. Rev. B 1992, 46, 16067-16080.

(6). Giannozzi, P.; Baroni, S.; Bonini, N.; Calandra, M.; Car, R.; Cavazzoni, C.; Ceresoli, D.; Chiarotti, G. L.; Cococcioni, M.; Dabo, I.; Dal Corso, A.; de Gironcoli, S.; Fabris, S.; Fratesi, G.; Gebauer, R.; Gerstmann, U.; Gougoussis, C.; Kokalj, A.; Lazzeri, M.; MartinSamos, L.; Marzari, N.; Mauri, F.; Mazzarello, R.; Paolini, S.; Pasquarello, A.; Paulatto, L.; Sbraccia, C.; Scandolo, S.; Sclauzero, G.; Seitsonen, A. P.; Smogunov, A.; Umari, P.; Wentzcovitch, R. M. QUANTUM ESPRESSO: a modular and open-source software project for quantum simulations of materials. J. Phys. Condens. Matter 2009, 21, 395502. (7). McMillan, W. L. Transition temperature of strong-coupled superconductors. Phys. Rev. 1968, 167, 331-344.

(8). Allen, P. B.; Dynes, R. C. Transition temperature of strong-coupled superconductors reanalyzed. Phys. Rev. B 1975, 12, 905-922.

(9). Giustino, F. Electron-phonon interactions from first principles. Rev. Mod. Phys. 2017, 89.

(10). Mostofi, A. A.; Yates, J. R.; Pizzi, G.; Lee, Y.-S.; Souza, I.; Vanderbilt, D.; Marzari, N. An updated version of wannier90: a tool for obtaining maximally-localised Wannier functions. Comput. Phys. Commun. 2014, 185, 2309-2310.

(11). Wu, Q.; Zhang, S.; Song, H.-F.; Troyer, M.; Soluyanov, A. A. WannierTools: an opensource software package for novel topological materials. Comput. Phys. Commun. 2018, $224,405-416$. 